\title{
Impact of Harmonic Power Terms on the Energy Measurement in AC Railways
}

\author{
Andrea Mariscotti, Senior Member, IEEE
}

\begin{abstract}
In electric ac railways a non-negligible amount of active and reactive power can be associated to distortion and harmonics. Electric railways are characterized by a mix of moving loads in quite variable operating conditions with a substantial superposition of different distortion patterns. The EN 50463-2 standard for the measurement of energy and of active and reactive power does not include harmonic and distortion power terms, leading to unavoidable underestimation. This work reports the estimate of the harmonic power for AC railways in real scenarios and its variability due to the operating conditions and moving loads, together with the uncertainty of the energy consumption estimate.
\end{abstract}

Keywords - Electric transportation systems, Energy consumption, Power Quality, Power system harmonics

\section{INTRODUCTION}

It is generally recognized that reactive power and harmonic distortion are responsible for increased losses and disturbance in distribution systems [1][2], from which the many Power Quality (PQ) standards, especially for LV and MV public and industrial networks. Similarly, in electrified traction systems losses may occur in the traction line and at substations. However, the study of power terms at fundamental and harmonics is also relevant for suitable and accurate estimate of power and energy consumption and fair billing. The attention is focused on ac railways being much more relevant than dc systems for distortion power, as demonstrated in [3]-[5]. Railways have been always considered a special "private" network with relatively immune loads as for harmonic distortion, so that harmonic limits were rarely stipulated for PQ purposes, but rather for the purpose of protecting signaling systems from interference [6][7] and in general to limit induced noise on nearby systems [8]. Energy consumption and fair billing aspects have become increasingly relevant, considering the efforts to improve energy efficiency especially for modern smart transportation systems [9]-[11] and the introduction of a complex normative for energy metering on-board [12]. Metering is achieved by measuring the electrical quantities at the train pantograph (voltage and current) [13]-[15].

The results here presented are developed in the framework of the 16ENG04 MyRailS Project and the European Union's Horizon 2020 research and innovation program. This work was supported by the Swiss State Secretariat for Education, Research and Innovation (SERI) under contract number 17.00127. The opinions expressed and arguments employed herein do not necessarily reflect the official views of the Swiss Government.

A. Mariscotti is with ASTM, Switzerland (andrea.mariscotti@astm-e.ch)
It is in general assumed that distortion components carry little active power and they are negligible. However, it will be demonstrated that for ac railways harmonic terms may carry in certain conditions a significant amount of active and non-active components and need suitable characterization.

The relevance of the pantograph harmonic power terms is evaluated not only in an absolute perspective (weighting the harmonic power with respect to the same quantities at the fundamental), but also taking into consideration the train operating conditions (acceleration, cruising, coasting, braking and standstill) and the rate of occurrence, determining in this way the influence on the overall exchanged energy (consumed and regenerated energy, to use the terminology of the EN 50463-2 [12]). The exchanged energy is estimated by means of an Energy Measurement Function (EMF) implemented onboard, that includes the data acquisition system and voltage and current sensors (the Voltage or Current Measurement Functions) and the Energy Calculation Function (ECF).

The EN 50463-2 [12], when specifying the limits of accuracy, clearly thinks in terms of nearly sinusoidal waveforms, with power and energy calculated taking into account only terms at the fundamental (see e.g. the indication of "maximum phase displacement at rated frequency"). Harmonics are then considered as a disturbance, from which the immunity requirements, common to the EN 50470-1 standard [16]. It is the objective of this work to demonstrate that neglecting the harmonic power terms may lead to errors in the total energy estimate of the same order of the EMF accuracy specification.

The definition of realistic conditions for the test of energy meters under non-sinusoidal conditions has attracted a lot of interest [17]-[19], but a clear solution has not been identified yet. The EN 50463-2 similarly does not clarify better the EMF and ECF for non-sinusoidal conditions, reflecting what was pointed out in [20] ten years ago: "The traditional quantities used for electrical energy pricing do not take into account the presence of harmonic distortion at the metering section. In fact, they are based upon the traditional concepts of active, reactive, and apparent power (and energy) and related power factor that are only well defined for sinusoidal voltages and currents."

The uncertainty of the ECF and EMF is thus influenced not only by the metrological characteristics of the VMF and CMF chains, but also by the adequate and all comprehensive definition of the objective quantities (power and energy) and their relationship with the basic quantities (pantograph voltage 
and current). As an input to standardization activities, the second objective is the definition of adequate measurement conditions, including the evaluation of the uncertainty due to the typical operating conditions and their variability.

The economic impact of a fractional energy saving with a magnitude commensurate to the observed variability and to the target uncertainty is definitely worth a better understanding of the problem. As pointed out in [3][4], using data from previous measurements and PQ assessment of some European railways, the magnitude of the identified harmonic power terms is indeed relevant for the uncertainty budget.

The following analysis is carried out in a single-train perspective, based on the pantograph voltage and current, as required for power and energy consumption assessment [3][4][12]. The measurement setup, instrumentation and uncertainty are described in [14]: the digitizer used 16 bits with a negligible impact; the Rogowskil coil and the capacitive voltage divider have the largest contribution of 3 and $1.4 \%$ $(k=2)$, respectively, accounting for all influencing factors, including noise, eccentricity, etc.

\section{POWER RELATED QUANTITIES}

Power quantities are defined using the IEEE Std. 1459 [22]. These definitions are well known and were already reported in the conference paper [3], of which this is an extension, so that they will be only briefly summarized for ease of reference.

The total apparent power in nonsinusoidal conditions $S$ is subdivided into active and non-active power terms. The identification of suitable terms to decompose the non-active term may be tricky as shown in [23][24]: what is relevant is separating the terms with non-null average from those with null average, the latter corresponding to distortion or reactive harmonic power. To this aim it is sufficient to calculate the total active power $P=P_{1}+P_{h}$ and then subtract it in a quadratic way from the total apparent power $S$, that can be conveniently calculated as the product of the rms values of voltage and current. The non-active power is then the Fryze's reactive power $Q_{h F}=\sqrt{S^{2}-P^{2}}$. The quantities at the fundamental $P_{1}$ and $Q_{1}$ are anyway calculated for exigency of comparison and normalization. The EN 50463-2 for ac railways, in fact, requires the measurement of active and reactive power in traction and braking conditions, and these quantities are defined based on the fundamental component only. We indicate them with obvious meaning as $P_{T}, P_{B}, Q_{T}$ and $Q_{B}$. In compliance with EN 50463-2 they are calculated when voltage and current satisfy some conditions, as detailed in sec. II.B.

This solves the problem of quantifying the overall contribution of harmonics to the active and reactive power, but not that of the relevance of single harmonic power terms.

For this reason the non-active power terms must be separated, using eq. (5) of the time-referred non-active power $p_{q}$ in [23], reported below:

- the first term is the harmonic reactive power $Q_{h}$;

- the second term is a set of mixed products defining the distortion power and involving both fundamental and harmonics (decomposing it further does not bring any useful information and reopen the discussion on the interpretation and the physical meaning of the terms);

- the last two terms are the contributions in presence of $\mathrm{dc}$ components, that in ac railways may be assumed zero, except during transients.

$$
\begin{aligned}
p_{q} & =-\sum_{h} V_{h} I_{h} \cos \vartheta_{h}\left[1-\cos \left(2 h \omega t-2 \alpha_{h}\right)\right]+ \\
& +2 \sum_{m}^{h} \sum_{m \neq n} V_{m} I_{n} \sin \left(m \omega t-\alpha_{m}\right) \sin \left(n \omega t-\beta_{n}\right)+ \\
& +V_{0} \sum_{h} I_{h} \sin \left(h \omega t-\beta_{h}\right)+I_{0} \sum_{h} V_{h} \sin \left(h \omega t-\alpha_{h}\right)
\end{aligned}
$$

The harmonic power terms are then expressed by means of convenient fractional indexes related to the fundamental:

- the index $k_{h}$ weights the active power carried by the $h$-th component $P_{h}(t)=\operatorname{Re}\left\{S_{h}(t)\right\}$ with respect to the active power at the fundamental $P_{1}(t): k_{h}=P_{h} / P_{1}$;

- the index $d_{h}$ measures instead the amount of active power with respect to the apparent power for the $h$-th component; assigning a positive sign to the incoming active power (i.e. absorbed by the train), it is possible to define a complex quantity of unitary modulus $d_{h}=P_{h} / S_{h}+j Q_{h} / S_{h}$, that can be conveniently displayed in polar coordinates.

These two quantities $\left(k_{h}\right.$ and $\left.d_{h}\right)$ may be expressed as a function of time (tracking during a train run) or as a function of the fundamental power terms themselves. They may be also grouped for a more compact representation, but this requires knowledge of the harmonic patterns and the characteristics of the on-board converters to be effective. A simple grouping based on frequency adjacency was used in [4][21] with the objective of identifying the frequency distribution of harmonic power and the necessary frequency extension to capture the relevant contributions. Various techniques, including correlation, may be used [3], to show the link with the operating conditions and to separate network distortion.

\section{A. Spectrum and harmonic power terms}

The spectra of $V_{p}$ and $I_{p}$ are calculated using the Short Time Fourier Transform (STFT) algorithm using two window lengths for the two different fundamental frequencies, $T_{16}$ and $T_{50}$. Frequency resolution is chosen so that even and odd harmonics are clearly detectable: $T_{16}=180 \mathrm{~ms}$ and $T_{50}=60 \mathrm{~ms}$ correspond to $1 / 3$ of the fundamental frequency. A Hann window is used for tapering. Usual time resolution to report power measurements is $1 \mathrm{~s}$; the EN 50463-2 requests energy consumption values every $5 \mathrm{~min}$.

\section{B. Harmonic power terms significance and selection}

The focus is on both active and non-active harmonic power terms, so harmonics are selected with the largest apparent power $S_{h}$. As known, in ac railways low-order harmonics are prevalent: to avoid that they bias heavily the selection, the largest harmonic terms are selected evenly in each subinterval 
of the frequency range, selected to focus both on networkrelated low-order harmonics, traction converter emissions and auxiliaries emissions [21]. The frequency range up to $5 \mathrm{kHz}$ is subdivided into three intervals, having selected $5 \mathrm{kHz}$ as a conveniently large upper frequency limit that includes the harmonic patterns of the on-board converters and their replica at multiples of the center frequency. The three intervals cover low (intv1, up to $500 \mathrm{~Hz}$ ), medium (intv2, between 500 and $1000 \mathrm{~Hz}$ ) and high frequency (intv3, between 1 and $5 \mathrm{kHz}$ ); for each interval the largest six $S_{h}$ are selected and processed, calculating $k_{h}$ and $d_{h}$ indexes.

Since the objective is providing information to the EN 50463-2, its criteria for power calculation are considered:

- "Area 1": full accuracy is required when $I_{p} \geq 10 \% I_{n}$; this accuracy is specified as the maximum error of the calculated energy and depends on the class, variable between 0.2 and $1 \%$; for $V_{p}<U_{\min 1}$ of EN 50163 [25], the required accuracy is as in Area 2;

- "Area 2": for $I_{p}$ between $1 \%$ and $10 \% I_{n}$ the required accuracy is relaxed by a factor of two;

- "Area 3": for $I_{p}$ between $0.4 \%$ and $1 \% I_{n}$ the energy measurement is required, but with unspecified accuracy;

- "Area 4": $I_{p}<0.4 \% I_{n}$ no energy measurement is really required (in Table 16 of EN 50463-2 we read "if energy is measured, no accuracy requirements"); as a consequence, no accuracy requirement is specified.

Similarly, the EN 50470-1 [16] specifies a transitional current $I_{t r}$ above which the "full accuracy requirements" apply up to the $I_{\max }$ threshold (above the nominal current $I_{n}$ with some margin), whereas between $I_{\min }$ and $I_{t r}$ "relaxed accuracy requirements" apply.

Based on these intervals the calculation of harmonic power terms will not be performed when the overall exchanged active power (absorbed or regenerated) is less than $0.1 \% P_{1 n}$, i.e. nominal fundamental power, in line with the accuracy requirements of the EN 50463-2. When processing very small quantities, in fact, much dispersed power index values may be observed, losing sometimes physical meaning (see results of $K_{H}$ at standstill in [3]).

\section{Uncertainty}

As known, operating conditions are quite variable and changes to the internal operation of the onboard converters may occur at any time, with significant influence on the resulting spectrum. The variability of the pantograph network impedance influences the way emissions are coupled into the network and the amount of distortion at the pantograph, as detailed in sec. III.C. For this reason it is reasonable in the following analysis to adopt margins in the selection of components and to report results using robust statistical figures. In addition, the train position and the relative displacement of other trains nearby exerts also its influence, by changing the way that internal and external components propagate and overlap [26]: although unlikely, it is also possible that components at the same frequency from different trains of the same model partially compensate, resulting in a lower amount of harmonic power at some frequencies.

For the uncertainty of the instrumentation used for the voltage, current and energy measurement functions (using the EN 50463-2 terminology), the $0.2 \%$ accuracy most stringent requirement of the EN 50463-2 is achievable. The selected $0.01 \% P_{1}$ significance threshold is also manageable considering that the experimental data are characterized by an overall uncertainty of $1-3 \%(k=2)$, including installation impact, but repeatability is an order of magnitude better [14]. A new measurement setup has been devised, assembled and installed as part of the "MyRailS" project [15][27], but the uncertainty assessment is not yet complete for comparison.

The uncertainty of data processing is that of FFT based methods, with an impact that is orders of magnitude smaller. Spectral leakage is controlled as described in sec. II.A.

\section{Tracking of harmonic power terms}

As anticipated, the most relevant harmonics will be tracked over a train run, correlating them to the overall operating conditions and in particular to the amount of exchanged power, assessing not only their variability and significance in relative terms, but also their contribution to the overall exchanged energy and their relevance as a systematic error of the EMF. In addition to the weighting of the $k_{h}$ and $d_{h}$ coefficients with respect to the overall power, time may be introduced to identify the cumulative effect of a single harmonic power term on the exchanged energy, telling what is the fractional change on average when that term is ignored.

\section{RAILWAY SYSTEMS DESCRIPTION}

An electrified railway may be visualized as a distribution network with multiple moving loads each characterized by significant dynamics and distortion [28]-[35]. The distribution occurs by means of the traction line that undergoes severe mechanical constraints and has thus a far from ideal electric behavior versus frequency [35]-[37]: voltage drops, resonance phenomena, induction, etc.

\section{A. $2 x 25 \mathrm{kV} 50 \mathrm{~Hz}$ system}

The traction line is fed with double-secondary transformers with their primary connected to high-voltage 3-phase lines; load is balanced by phase rotation, tapping cyclically different pairs of phases. This arrangement requires the electrical isolation by phase separation sections of adjacent line sections, each fed by one substation. For the considered Italian highspeed line case, the installed power is quite large (electric substations are rated $60 \mathrm{MVA}$, autotransformers $15 \mathrm{MVA}$ ) and the length of supply sections is in the order of $25-30 \mathrm{~km}$.

\section{B. $15 \mathrm{kV} 16.7 \mathrm{~Hz}$ system}

The $15 \mathrm{kV} 16.7 \mathrm{~Hz}$ system has a highly interconnected network with supply sections longer than those of modern $2 \times 25$ $\mathrm{kV} 50 \mathrm{~Hz}$ systems [21]. This increases the chance of network resonances already at low frequency, causing in some cases the amplification of voltage or current distortion [14]. The $16.7 \mathrm{~Hz}$ 
network is also used for mixed traffic (long/medium distance and commuter traffic).

\section{Line resonances and anti-resonances}

The pantograph line impedance is subject to a significant variability versus frequency and longitudinal position of the train [35][36], as shown in Fig. 1. The influence on current and voltage distortion and on harmonic power terms can be explained with an example. A loco pulls some traction current $I_{p}$ characterized by a few harmonics under quite large pantograph impedance near a resonance, so that voltage distortion is increased. Symmetrically, at an anti-resonance with particularly low values of pantograph impedance the $I_{p}$ current components are "amplified" in a situation of nearly short-circuit condition at those frequencies. In both situations the product of the line voltage and rolling stock current will increase, giving rise to an increase of the harmonic power terms. In addition, at resonances and anti-resonances the impedance of the traction line becomes almost resistive [37], increase significantly the amount of harmonic active power.

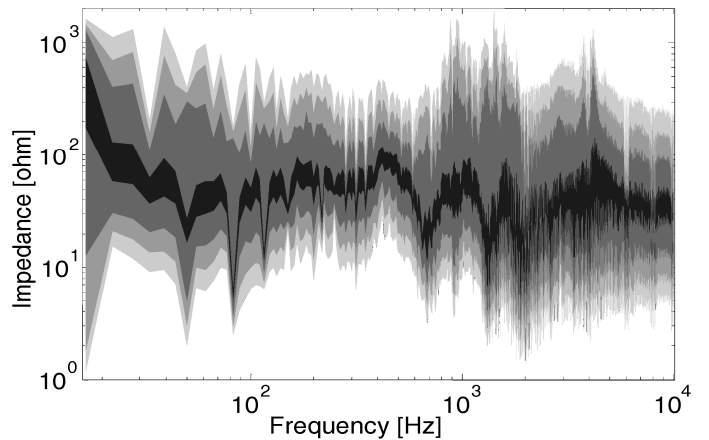

Fig. 1. Example of pantograph line impedance variability using histogram curve (5-20-40-60-80-95\%) [14].

\section{RESUlTS FOR 15 KV 16.7 HZ AND 25 KV 50 HZ SYSTEMS}

Results are reported for one $16.7 \mathrm{~Hz}$ and one $50 \mathrm{~Hz}$ system. An instrumented train ran in normal commercial service for several $\mathrm{km}$ and data have been extracted for both the identification of typical significant events and phenomena, and to derive statistical figures based on the entire ensemble. The amount of data comes from about two days of continuous recording for each railway system. The results are as follows:

1) typical run showing power absorption and regeneration profiles (train dynamics) and the calculated output quantities as per EN 50463-2 for both the fundamental and harmonic terms up to $5 \mathrm{kHz}$;

2) identification of the most relevant harmonic power terms setting and tracking the intv 1 to intv 3 groups of sec. II.B; the stability of the membership is also checked;

3) for the selected components belonging to a group indexes $k_{h}$ and $d_{h}$ are calculated and displayed for a test run, as well as their statistics are given for the entire set of data;
4) the impact on the energy measurement uncertainty is evaluated considering the output quantities at step 1) and the statistics at step 3).

\section{A. Run profiles, spectra and EN 50463-2 quantities}

Two typical profiles are shown in Fig. 2, where a sequence of traction, cruising/coasting and braking phases may be seen, including time intervals with stops at stations.

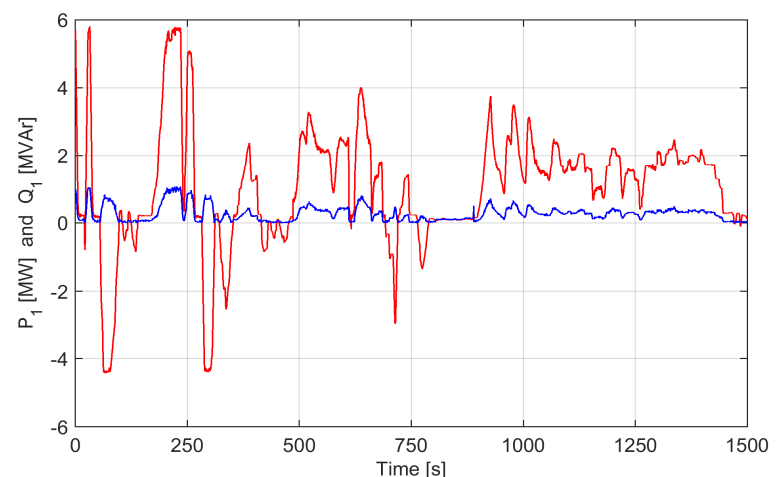

(a)

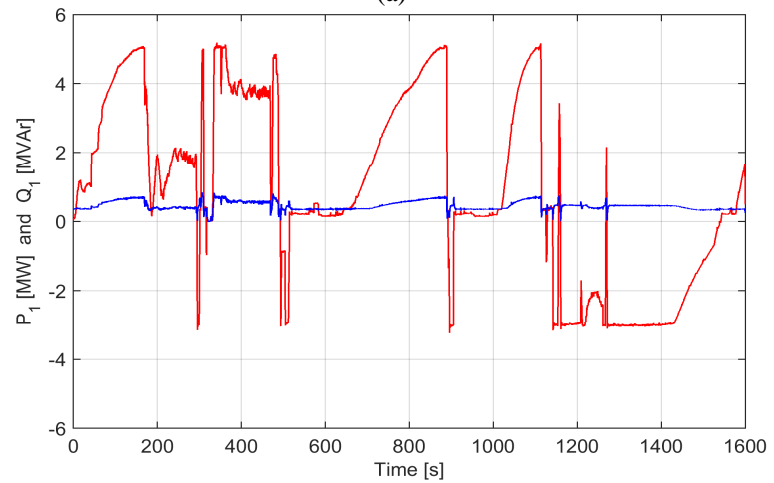

(b)

Fig. 2. Fundamental active $P_{1}$ (red) and reactive $Q_{1}$ (blue) power for (a) 15 $\mathrm{kV} 16.7 \mathrm{~Hz}$ and (b) $2 \times 25 \mathrm{kV} 50 \mathrm{~Hz}$ test run.

Samples of the typical line spectra with visible odd characteristic harmonics are shown in Fig. 3, where a good correspondence with the three intervals intv 1 to intv 3 may be noted. The low frequency components of intv1 are in general related to network distortion; the clusters of spectral lines at higher frequency are the patterns of the on-board converters, mostly of the four-quadrant type. The particularly intense third harmonic $(50 \mathrm{~Hz})$ in the $16.7 \mathrm{~Hz}$ system is due to the on-board auxiliaries working at $50 \mathrm{~Hz}$, overlapping to the 3rd harmonic of the fundamental [3].

During the visual inspection of data an excessive distortion was observed, not justified by the interaction of the train with a line resonance for two reasons: it lasted for a very long time and it was located at $550 \mathrm{~Hz}$ (11th harmonic), so well below the first line resonance of a $2 \times 25 \mathrm{kV}$ system. The harmonic is well visible in Fig. 3. The energy parameters in sec. IV.C are thus calculated for the whole test run and excluding the first portion up to time $327 \mathrm{~s}$, where the neutral section is located. As commented below Table III there is no significant impact, however, on the behavior of the energy values. 

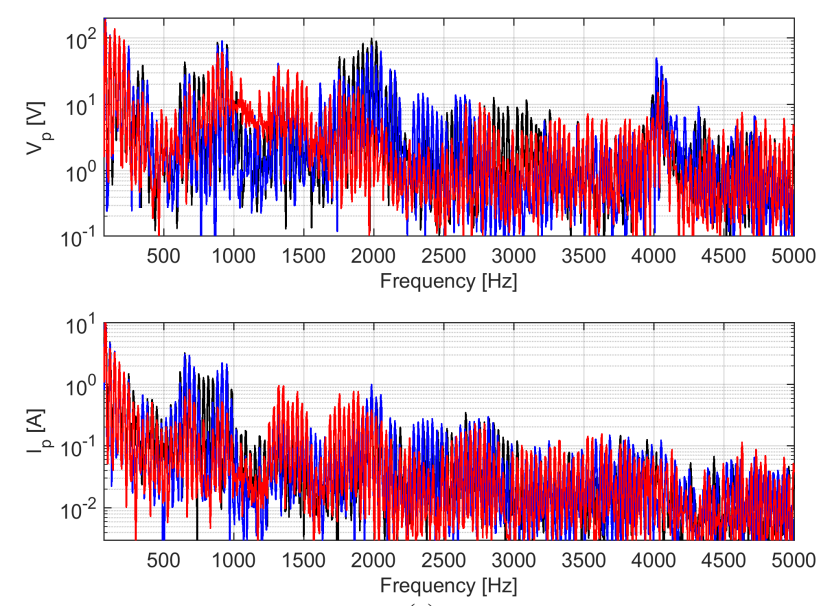

(a)
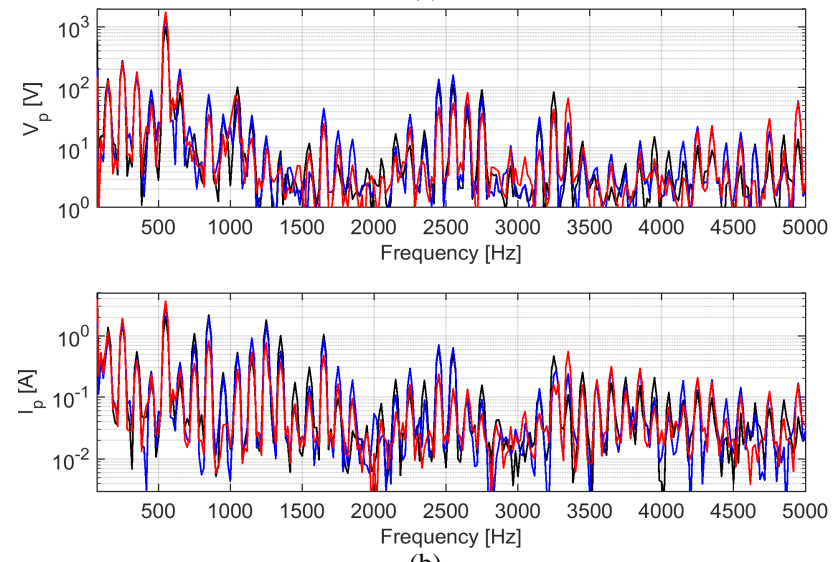

(b)

Fig. 3. Harmonic active power $P_{h}$ vs. frequency and time in $\mathrm{dB}\left(10 \log _{10}\right)$ : (a) $15 \mathrm{kV} 16.7 \mathrm{~Hz}$ and (b) $2 \times 25 \mathrm{kV} 50 \mathrm{~Hz}$ test run.

\section{B. Harmonic power groups and tracking $k_{h}$ and $d_{h}$}

As outlined in sec. II, STFT spectra were processed to extract the intv 1 to intv 3 groups, based on the magnitude of the harmonic apparent power $S_{h}$. The $k_{h}$ and $d_{h}$ indexes for the most significant harmonics from the previous selection are then tracked versus time. The absolute value of $k_{h}$ is shown in Fig. 4 using a logarithmic axis: the $3 \mathrm{rd}$ harmonic is responsible for a significant fraction of the total harmonic active power; although some low-order harmonics give the largest contribution (namely the 3rd and the 5th), the value of $\mathrm{Ph}$ is quite well distributed among the various harmonic clusters around the characteristic harmonics of the traction converters and auxiliaries. The evolution of the three selected harmonics versus time is shown in Fig. 5, where the behavior is quite regular as made it clear in the histogram.

The power flow and the inductive/capacitive behavior is analyzed plotting the index $d_{h}$ in polar coordinates, against the absolute value of $\mathrm{P}_{1}$ (see Fig. 6). It is possible to observe a reversal of some components (such as 119th and 121st know to belong to the harmonic pattern of the locomotive converter, and the 7 th, for which the explanation is not easy); conversely the 5th, 9th, 41st, 43rd and 45th are clearly uninfluenced and caused by an external source.
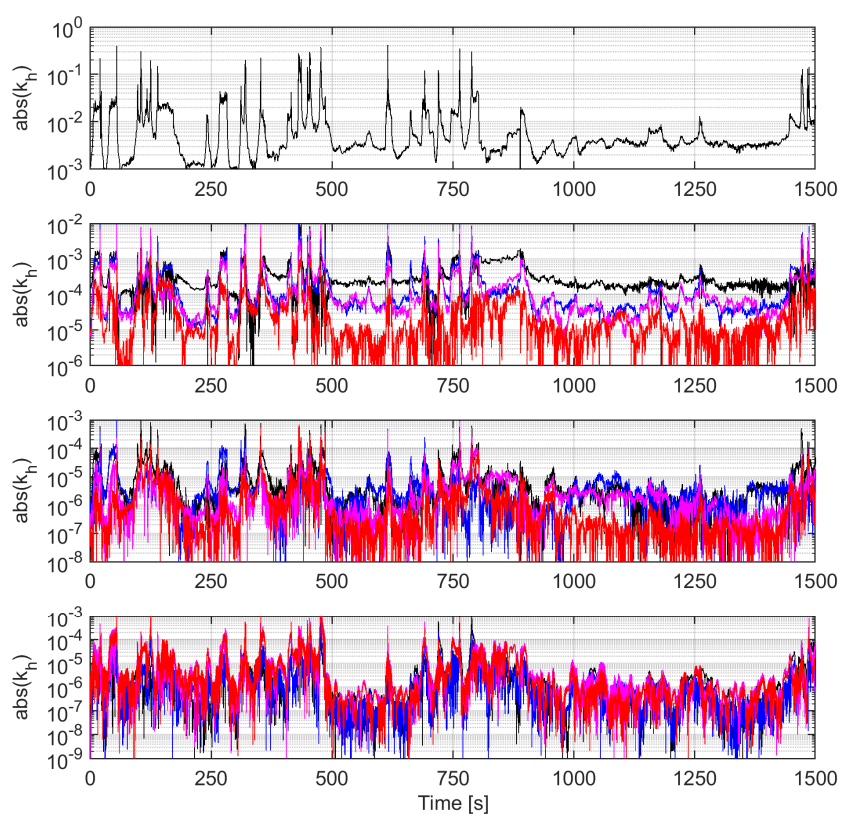

Fig. 4. Tracking of $k_{h}$ (from top to bottom): 1) 3rd, 2) 5th (black), 7th blue), 9th (magenta) and 11th (red); 3) 39th (black), 41st (blue), 43rd (magenta), 45th (red); 4) 115th (black), 117th (blue), 119th (magenta), 121st (red).
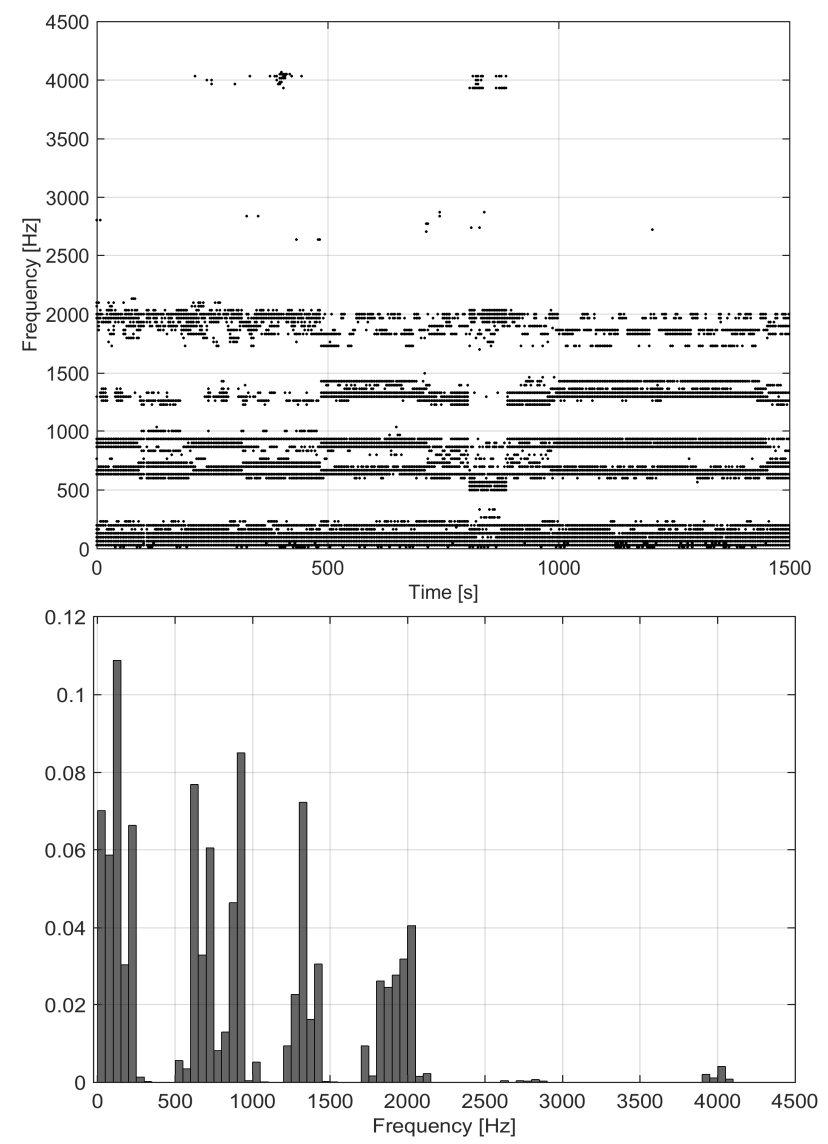

Fig. 5. Membership plot of selected harmonic power terms for the $16.7 \mathrm{~Hz}$ system: characteristic harmonics of converters are clearly visible. 

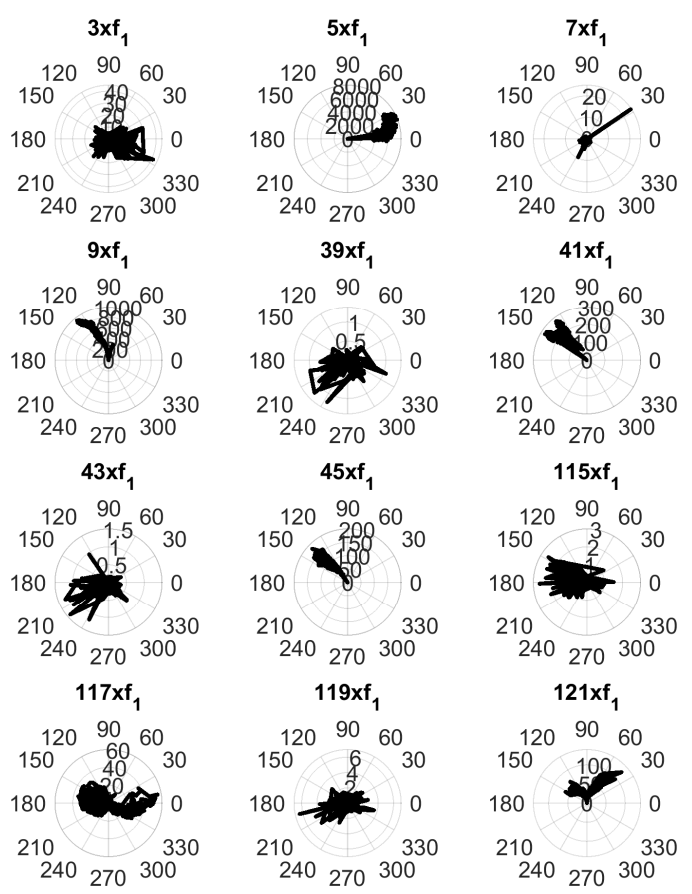

$115 \mathrm{xf}_{1}$
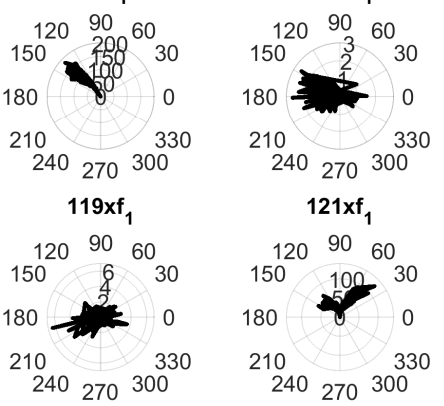

(a)

$3 \mathrm{xf}_{1}$

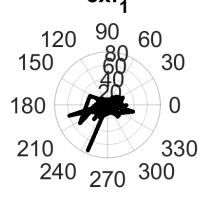

$5 \mathrm{xf}_{1}$

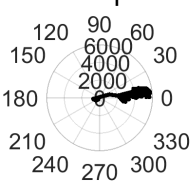

$39 \mathrm{xf}_{1}$

9xf

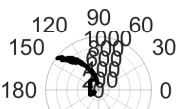

$\begin{array}{ll}210 & 330 \\ 240 & 270 \\ 300\end{array}$

$$
43 \mathrm{xf}_{1}
$$

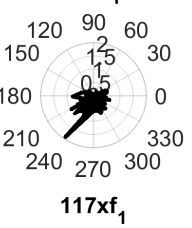

$2090 \quad 60$

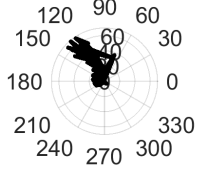

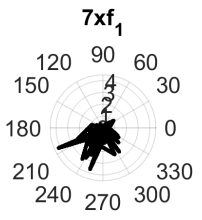

$41 \mathrm{xf}_{1}$

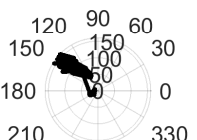

$\begin{array}{cr}210 & 330 \\ 240 \quad 270 & 300\end{array}$

$115 x_{1}$
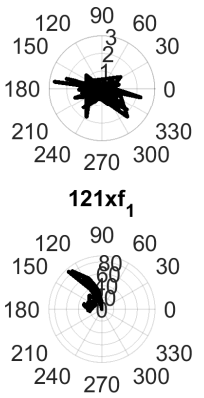

210330

Fig. 6. Polar plot of $P_{h}-Q_{h}$ angle against the traction effort (absolute value of $\left.P_{1}\right)$ distinguishing (a) acceleration $\left(P_{1}>0\right)$ and (b) braking $\left(P_{1}<0\right)$.

\section{Impact on the energy measurement uncertainty}

The energy values of the runs in Fig. 2 are calculated distinguishing traction and braking phases and active and reactive power, including in the latter the distortion terms discussed in sec. II. Energy is calculated simply as the sum of the power terms (active or reactive) in each phase (traction or

braking), and multiplying for the time interval between sampled power values (that corresponds to $T_{16}$ and $T_{50}$ ). The use of the term "energy" is an abuse for reactive power, but allows a uniform notation in line with that of EN 50463-2: the unit of measure will be Joule.

TABLE I. ENERGY VALUES AND ERROR (15 KV $16.7 \mathrm{~Hz})$

\begin{tabular}{|c|c|c|c|c|}
\hline Quantity & Area 1 & Area 2 & Area 3 & Area 4 \\
\hline $\mathrm{E}_{\mathrm{AT}}[\mathrm{MJ}]$ & 1878.3 & 81.513 & 0.4925 & 0.02383 \\
$\Delta \mathrm{E}_{\mathrm{AT}}[\mathrm{kJ}]$ & 4.6049 & 0.9059 & 0.04238 & 0.004189 \\
err \% & $0.25 \%$ & $1.11 \%$ & $8.61 \%$ & $17.6 \%$ \\
\hline $\mathrm{E}_{\mathrm{AB}}[\mathrm{MJ}]$ & -325.76 & -33.664 & -3.1726 & -0.03311 \\
$\Delta \mathrm{E}_{\mathrm{AB}}[\mathrm{kJ}]$ & 0.5644 & 0.3209 & 0.02350 & 0.004782 \\
err \% & $-0.17 \%$ & $-0.95 \%$ & $-0.74 \%$ & $-14.4 \%$ \\
\hline $\mathrm{E}_{\mathrm{RT}}[\mathrm{MJ}]$ & 330.83 & 26.078 & 0.5973 & 0.03075 \\
$\Delta \mathrm{E}_{\mathrm{RT}}[\mathrm{kJ}]$ & 244.08 & 62.478 & 3.2326 & 0.4354 \\
err \% & $73.8 \%$ & $239.6 \%$ & $541.2 \%$ & $1415.9 \%$ \\
\hline $\mathrm{E}_{\mathrm{RB}}[\mathrm{MJ}]$ & 49.398 & 2.8379 & 0.1473 & 0.02735 \\
$\Delta \mathrm{E}_{\mathrm{RB}}[\mathrm{kJ}]$ & 32.425 & 19.018 & 1.587 & 0.4207 \\
err \% & $65.6 \%$ & $670.1 \%$ & $1077.4 \%$ & $1538.2 \%$ \\
\hline
\end{tabular}

TABLE II. ENERGY VALUES AND ERROR (2X25 KV 50 HZ)

\begin{tabular}{|c|c|c|c|c|}
\hline Quantity & Area 1 & Area 2 & Area 3 & Area 4 \\
\hline $\mathrm{E}_{\mathrm{AT}}[\mathrm{MJ}]$ & 2407.1 & 230.56 & 0.07933 & 0.01063 \\
$\Delta \mathrm{E}_{\mathrm{AT}}[\mathrm{kJ}]$ & -200.40 & 67.059 & -0.0604 & 0 \\
err \% & $-8.24 \%$ & $29.1 \%$ & $-76.1 \%$ & $0 \%$ \\
\hline $\mathrm{E}_{\mathrm{AB}}[\mathrm{MJ}]$ & -1007.7 & -55.161 & 0 & 0 \\
$\Delta \mathrm{E}_{\mathrm{AB}}[\mathrm{kJ}]$ & -159.21 & 4.259 & 0 & 0 \\
err \% & $15.8 \%$ & $-7.7 \%$ & - & - \\
\hline $\mathrm{E}_{\mathrm{RT}}[\mathrm{MJ}]$ & 372.59 & 195.82 & 0.2732 & 0.06089 \\
$\Delta \mathrm{E}_{\mathrm{RT}}[\mathrm{MJ}]$ & 84.577 & 32.551 & 0.2253 & 0.05547 \\
err \% & $22.7 \%$ & $16.6 \%$ & $82.5 \%$ & $91.1 \%$ \\
\hline $\mathrm{E}_{\mathrm{RB}}[\mathrm{MJ}]$ & 156.83 & 23.129 & 0 & 0 \\
$\Delta \mathrm{E}_{\mathrm{RB}}[\mathrm{MJ}]$ & 31.940 & 4.1008 & 0 & 0 \\
err \% & $20.4 \%$ & $17.7 \%$ & - & - \\
\hline
\end{tabular}

TABLE III. ENERGY VALUES AND ERROR (2X25 KV $50 \mathrm{~Hz}$, AFTER NEUTRAL SECTION TIME $>327 \mathrm{~s}$ )

\begin{tabular}{|c|c|c|c|c|}
\hline Quantity & Area 1 & Area 2 & Area 3 & Area 4 \\
\hline $\mathrm{E}_{\mathrm{AT}}[\mathrm{MJ}]$ & 1696.1 & 158.81 & 0.01785 & 0.01063 \\
$\Delta \mathrm{E}_{\mathrm{AT}}[\mathrm{kJ}]$ & -201.40 & 31.553 & 0.02344 & 0 \\
err \% & $-11.9 \%$ & $19.1 \%$ & $131.3 \%$ & $0 \%$ \\
\hline $\mathrm{E}_{\mathrm{AB}}[\mathrm{MJ}]$ & -991.46 & -54.179 & 0 & 0 \\
$\Delta \mathrm{E}_{\mathrm{AB}}[\mathrm{kJ}]$ & -161.06 & 3.4416 & 0 & 0 \\
err \% & $16.2 \%$ & $-6.4 \%$ & - & - \\
\hline $\mathrm{E}_{\mathrm{RT}}[\mathrm{MJ}]$ & 257.59 & 163.69 & 0.1456 & 0.06089 \\
$\Delta \mathrm{E}_{\mathrm{RT}}[\mathrm{MJ}]$ & 49.868 & 24.929 & 0.1277 & 0.05547 \\
err \% & $19.4 \%$ & $15.2 \%$ & $87.7 \%$ & $91.1 \%$ \\
\hline $\mathrm{E}_{\mathrm{RB}}[\mathrm{MJ}]$ & 154.72 & 22.637 & 0 & 0 \\
$\Delta \mathrm{E}_{\mathrm{RB}}[\mathrm{MJ}]$ & 31.092 & 3.9449 & 0 & 0 \\
err \% & $20.1 \%$ & $17.4 \%$ & - & - \\
\hline
\end{tabular}

A few observations may be made observing the calculated energy values and the correction when all harmonic terms are included:

- the error caused by the harmonic correction is larger for areas 3 and 4 with lower accuracy, belonging to lower rms current values; area 2 accounts approximately for 5$10 \%$ of the active power, much variable for reactive energy, and also when comparing the two systems; 
- deviations for the "active energy" are much smaller, as expected, as the harmonics carry a moderate amount of power;

- the two systems behave quite differently, in that for the $2 \times 25 \mathrm{kV} 50 \mathrm{~Hz}$ despite the better harmonic distortion, the harmonics carry much more active power;

- the amount of reactive energy increases significantly if the harmonic non-active terms are included; although there is no direct relationship with energy consumption, they indicate higher system losses;

- regarding the higher distortion before the neutral section, it may be said that the changes in the energy consumption parameters are not dramatic, but a $10 \%$ reduction of EAT in area 2 may be observed.

In general, the deviations in the calculated energy are significant and represent a significant uncertainty for the EMF in terms of definition of terms. Harmonic power and energy should be analyzed more deeply with a much wider set of data, to increase the significance of results and to avoid biasing the judgment with local phenomena and singular cases.

\section{CONCLUSIONS}

The behavior of the harmonic active and non-active power terms has been considered for two railway systems, a $15 \mathrm{kV}$ 16.7 $\mathrm{Hz}$ and a $2 \times 25 \mathrm{kV} 50 \mathrm{~Hz}$ system, extending the analysis presented in [3]. The objective is the analysis of the typical distortion patterns in ac railways and the verification of the relevance of such terms to the Energy Measurement Function of the EN 50463-2 standard. To this aim the most relevant harmonic power terms have been identified and tracked over train runs longer than 25 minutes each: they clearly depend on the harmonic patterns of the on-board converters, possibly caused by trains nearby and by the network, but consistently present along the entire run, except in situations where major transients occur or auxiliaries predominate (stops at stations).

The target is the procedure of the EN 50463-2 and the EMF uncertainty with respect to the influence of factors (the harmonic power terms) not included in the approach indicated in the standard. It is shown that the exclusion of the harmonic power terms from the computation causes errors of the estimate which may be significant. Calculating energy based only on the quantities at the fundamental $\left(P_{1}\right.$ and $\left.Q_{1}\right)$ does not necessarily underestimate, compared to the result obtained including all harmonic power terms up to $5 \mathrm{kHz}$, as considered here.

\section{REFERENCES}

[1] G.W. Massey, "Estimation Methods for Power System Harmonic Effects on Power Distribution Transformers," IEEE Trans. on Industry Applications, Vol. 30, n. 2, Mar/Apr. 1994, pp. 485-489.

[2] A.E. Emanuel and X. Wang, "Estimation of Loss of Life of Power Transformers Supplying Nonlinear Loads," IEEE Trans. on Power Apparatus and Systems, Vol. 104, n. 3, March 1985, pp. 628-636.

[3] A. Mariscotti, "Behaviour of Spectral Active Power Terms for the Swiss 15 kV 16.7 Hz Railway System," Proc. of 10th IEEE Intern. Workshop on Applied Measurements for Power Systems, Sept. 25-27, 2019, Aachen, Germany.
[4] A. Mariscotti, "Characterization of Active Power Flow at Harmonics for AC and DC Railway Vehicles," Proc. of the IEEE Vehicle Power and Propulsion Conf., Oct. 14-17, 2019, Hanoi, Vietnam.

[5] H. Douglas, C. Roberts, S. Hillmansen and F. Schmid, "An assessment of available measures to reduce traction energy use in railway networks," Energy Convers. Manage., Vol. 106, 2015, pp. 1149-1165.

[6] A. Mariscotti, M. Ruscelli and M. Vanti, "Modeling of Audiofrequency Track Circuits for validation, tuning and conducted interference prediction", IEEE Trans. on Intelligent Transp. Systems, vol. 11, n. 1, March 2010, pp. 52-60.

[7] L. Zhao and M. Li, "Probability Distribution Modeling of the Interference of the Traction Current in Track Circuits," J. of Theoretical and Applied Inform. Techn., Vol. 46, n. 1, 2012, pp. 125-131.

[8] J. Bongiorno, G. Boschetti, and A. Mariscotti, "Low-Frequency Coupling: Phenomena in Electric Transportation Systems", IEEE Electrification Magazine, Sept. 2016, Vol. 4, no. 3, pp. 15-22.

[9] G. Hinrichs and J. Hegarty, "Introduction of energy metering, settlement and billing at SBB," European Railway Review, Vol. 22 n. 1, 2016 (online https://www.globalrailwayreview.com/article/26308/introduction-ofenergy-metering-settlement-and-billing-at-sbb/)

[10] A. González-Gil, R. Palacin, P. Batty, and J.P. Powell, “A systems approach to reduce urban rail energy consumption," Energy Conversion and Management, Vol. 80, 2014, pp. 509-524.

[11] D. Giordano et al., "Accurate measurements of energy, efficiency and power quality in the electric railway system," Conf. on Precision Electromagnetic Measurements (CPEM), July 8-13, 2018, Paris, France.

[12] CENELEC EN 50463-2, Railway applications - Energy measurement on board trains, 2017.

[13] A. Delle Femine, D. Gallo, C. Landi and M. Luiso, "Discussion on DC and AC Power Quality Assessment in Railway Traction Supply Systems," Proc. of the IEEE Intern. Instrum. and Meas. Technology Conf., May 20-23, 2019, Auckland, New Zealand.

[14] A. Mariscotti, "Direct Measurement of Power Quality over Railway Networks with Results of a $16.7 \mathrm{~Hz}$ Network," IEEE Trans. on Instrum. and Meas., vol. 60 n. 5, May 2011, pp. 1604-1612.

[15] G. Crotti et al., "Monitoring Energy and Power Quality On Board Train," Proc. of 10th IEEE Intern. Workshop on Applied Meas. for Power Systems, Sept. 25-27, 2019, Aachen, Germany.

[16] CENELEC EN 50470-1, Electricity metering equipment (a.c.) - Part 1: General requirements, tests and test conditions - Metering equipment (class indexes A, B and C), 2018.

[17] A. Olencki and P. Mroz, "Testing of energy meters under three-phase determined and random nonsinusoidal conditions," Metrol. and Meas. Syst., Vol. 21, n. 2, 2014, pp. 217-232.

[18] L.T.S. Oliveira, R.F.B. de Oliveira, J.R. Macedo and G. Leal, "Performance analisys of active energy meters in non-sinusoidal conditions," proc. of 2018 Simposio Brasileiro de Sistemas Eletricos (SBSE), May 12-16, 2018, Niteroi, Brazil.

[19] A. Cataliotti, V. Cosentino, A. Lipari and S. Nuccio, "Metrological Characterization and Operating Principle Identification of Static Meters for Reactive Energy: An Experimental Approach Under Nonsinusoidal Test Conditions," IEEE Trans.on Instrum. and Meas., Vol. 58, n. 5, May 2009, pp. 1427-1435.

[20] A. Cataliotti and V. Cosentino, "Disturbing loads identification in power systems: a single-point time-domain method based on the IEEE 14592000," IEEE Trans.on Instrum. and Meas., Vol. 58, n. 5, May 2009, pp. 1436-1445.

[21] A. Mariscotti, "Relevance of Harmonic Active Power Terms for Energy Consumption in some Railway Systems," in Proc. of the 24th IMEKO TC4 Int. Symp., Sept. 17-20, 2019, Xi'an, China.

[22] IEEE Std. 1459, IEEE Standard Definitions for the Measurement of Electric Power Quantities under Sinusoidal, Nonsinusoidal, Balanced, or Unbalanced Condition, 2010.

[23] P.V. Barbaro, A. Cataliotti, V. Cosentino and S. Nuccio, "A Novel Approach Based on Nonactive Power for the Identification of Disturbing Loads in Power Systems," IEEE Trans. on Power Delivery, Vol. 22, n. 3, July 2007, pp. 1782-1789. 
[24] A. E. Emanuel, "Powers in nonsinusoidal situations - A review of definitions and physical meaning," IEEE Trans. on Power Delivery, Vol. 5, n. 3, July 1990, pp. 1377-1389.

[25] CENELEC EN 50163, Railway applications - Supply voltages of traction systems, 2013.

[26] B. Hemmer, A. Mariscotti, D. Wuergler, "Recommendations for the calculation of the total disturbing return current from electric traction vehicles", IEEE Trans. on Pow. Del., Vol. 19, n. 2, Apr. 2004, pp. 11901197.

[27] A. Delle Femine, D. Gallo, C. Landi and M. Luiso, "Discussion on DC and AC Power Quality Assessment in Railway Traction Supply Systems," Proc. of the IEEE Intern. Instrum. and Meas. Technology Conf., May 20-23, 2019, Auckland, New Zealand.

[28] M. Tanta et al., "Power quality phenomena in electrified railways: Conventional and new trends in power quality improvement toward public power systems," Proc. of the Intern. Young Engineers Forum, May 4, 2018, Lisbon, Portugal.

[29] D. Serrano-Jiménez, L. Abrahamsson, S. Castaño-Solís and J. SanzFeito, "Electrical railway power supply systems: Current situation and future trends," Intern. J. of Electrical Power \& Energy Systems, Vol. 92, Nov. 2017, pp. 181-192.

[30] A. Dolara, M. Gualdoni and S. Leva, "Impact of High-Voltage Primary Supply Lines in the $225 \mathrm{kV}-50 \mathrm{~Hz}$ Railway System on the Equivalent Impedance at Pantograph Terminals," IEEE Trans. on Power Delivery, Vol. 27, n. 1, Jan. 2012, pp. 164-175.
[31] M. Brenna, F. Foiadelli, M. Roscia and D. Zaninelli, "Current Distortion Evaluation in Traction 4Q Constant Switching Frequency Converters," Journal of Electromagnetic Analysis and Applications, Vol. 3, n. 3, Jan. 2009, pp. 129-137.

[32] P. Pan, H. Hu, X. Yang, F. Blaabjerg, X. Wang and Z. He, "Impedance Measurement of Traction Network and Electric Train for Stability Analysis in High-Speed Railways," IEEE Trans. on Power Electronics, Vol. 33, n. 12, Dec. 2018, pp. 10086-10100.

[33] S. Gao, X. Li, X. Ma, H. Hu, Z. He and J. Yang, "Measurement-Based Compartmental Modeling of Harmonic Sources in Traction PowerSupply System," IEEE Trans. on Pow. Del., Vol. 32, n. 2, Apr. 2017, pp. 900-909.

[34] K. D. Lee, S. B. Leeb, L. K. Norford, P. R. Armstrong, J. Holloway and S. R. Shaw, "Estimation of Variable-Speed-Drive Power Consumption from Harmonic Content," IEEE Trans. on Energy Conv., Vol. 20, n. 3, Sept. 2005, pp. 566-574.

[35] J. Holtz and H.J. Klein, "The propagation of harmonic currents generated by inverter fed locomotives in the distributed overhead supply systems," IEEE Trans. on Pow. Electr., Vol. 4, n. 2, Apr. 1989, pp. 168-174.

[36] M. Fracchia, A. Mariscotti, P. Pozzobon, "Track and traction line impedance expressions for deterministic and probabilistic voltage distortion analysis", IEEE Intern. Conf. on Harm. and Quality of Power ICHQP, Orlando, FL, USA, Oct. 21-25, 2000, pp. 589-594.

[37] A. Mariscotti, P. Pozzobon, "Synthesis of line impedance expressions for railway traction systems," IEEE Trans. on Vehicular Technology, vol. 52 n. 2, March 2003, pp. 420-430. 